\title{
Theory of Light Scattering by an Isotropic System Composed of Anisotropic Units. Out-of-Plane Scattering
}

\author{
Kazuo NAGAI \\ Government Industrial Research Institute, Osaka, \\ Midorigaoka 1, Ikeda, Osaka, Japan.
}

(Received December 18, 1971)

\begin{abstract}
A previous theory [Polymer J., 3, 67 (1972)] of light scattering by an isotropic system composed of anisotropic units is extended to the case of the out-ofplane scattering where the intensity of scattered light is measured outside the scatteringplane. Two cases are considered: the intensity is measured photoelectrically in one and photographically in the other. It is shown that the out-of-plane scattering at any direction can be reduced to the in-plane scattering with the planes of polarization of the polarizer and the analyzer inclined properly (by $\psi$ and $\omega$ ) to the vertical direction. In the photoelectric case the intensity $I(\psi, \omega)$ of the latter scattering can be expressed as $I(\psi, \omega)=\tilde{a} I_{\mathrm{Vv}}+\tilde{b} I_{\mathrm{Hv}}+\tilde{c} I_{\mathrm{Hh}}+\tilde{d} I\left(45^{\circ}, 45^{\circ}\right)$, where $I_{\mathrm{HV}} \equiv I\left(0^{\circ}, 90^{\circ}\right)$ etc. $I\left(45^{\circ}, 45^{\circ}\right)$ (or some alternative) is found to give information substantially independent of those which were previously studied on $I_{\mathrm{Vv}}, I_{\mathrm{Hv}}$, and $I_{\mathrm{Hh}}$. A refined method for analysis of experimental data is proposed on the basis of the four intensity functions described above. In the photographic case three photographs are shown to give all of the information, which are taken with such experimental arrangements that the plane of polarization of the polarizer is set vertical while that of the analyser is set vertical, ( $\mathbf{v}$ pattern) or horizontal (Hv pattern).
\end{abstract}

KEY WORDS Light Scattering / Isotropic System / Anisotropic Units / Out-of-Plane Scattering / General Polymer Chain / PorodKratky Chain / Photoelectric Case / Photographic Case /

In a previous paper $^{1}$ we developed a theory of light scattering by an isotropic system composed of anisotropic units. The treatment was restricted to the case of the in-plane scattering where the intensity of scattered light is measured in the scattering plane (the $x y$ plane of Figure

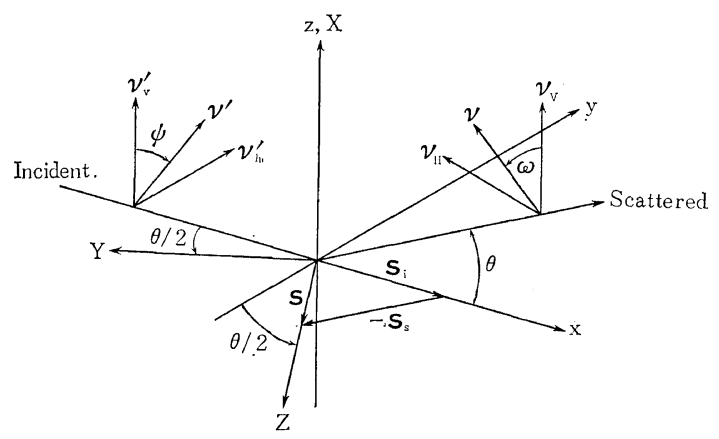

Figure 1.
1) while the incident and scattered lights are vertically or horizontally polarized with respect to the scattering plane. This corresponds to the classical experimental arrangement for investigations of polymer solutions. On the occasion of the U. S.-Japan Seminar on Statistical Mechanics and Spectroscopy of Polymers (University of Massachusetts, Amherst, Mass., August 2-6, 1971) Prins suggested that the out-of-plane scattering should yield more information than the in-plane scattering on a scattering system (see Gouda and Prins ${ }^{2}$ ). Therefore, in this paper we have extended the previous treatment to this more general case. We examined two cases: the intensity is measured photoelectrically in one and photographically in the other, emphasis being laid on the former.

The previous paper ${ }^{1}$ will hereafter be referred to as I and equation $a b$ of that paper as eq I- 
ab. Symbols defined in I will be used without definition (see Glossary of Principal Symbols of I). The reader is hoped to have read I.

\section{CASE OF PHOTOELECTRIC DETECTION}

\section{General Out-of-Plane Scattering}

In this case the intensity of out-of-plane scattered light is measured with a photoelectric tube with its optical axis set parallel to the scattered beam. The vertically polarized incident light, i.e., the case of $\nu_{\mathrm{v}}{ }^{\prime}$ in Figure 1, is usually employed. As is well known and can readily be shown, the out-of-plane scattering can be reduced to the in-plane scattering if $\boldsymbol{\nu}^{\prime}$ and $\nu$ are properly chosen. To see this, first consider a case of the in-plane scattering in which the planes of polarization of the polarizer and the analyzer make angles $\phi$ and $\omega$, respectively, with the vertical $z$ direction, as shown in Figure 1. The out-of-plane scattering is realized by rotating the light scattering framework-polarizer, sample, analyzer, and photoelectric tube-about the $x$ axis by angle $\psi$. Of course the equivalence is valid only when a sample is isotropic as a whole, and it is such an isotropic system to which I and this paper are exclusively devoted. Namely the out-of-plane scattering with any polarizations of the incident and scattered lights can be reduced to the in-plane scattering with proper $\psi$ and $\omega$, so it suffices to consider the latter case. The previous formulation ${ }^{1}$ is readily adaptable to this case. In this paper we call the in-plane scattering with $\phi, \omega \neq 0$ or multiples of $90^{\circ}$ as the out-of-plane scattering.

$\nu^{\prime}$ and $\boldsymbol{\nu}$ are given by $(0, \sin \psi, \cos \psi)^{T}$ and $(-\sin \omega \sin \theta, \sin \omega \cos \theta, \cos \omega)^{T}$ in the $x y z$ system, and therefore ( $c f$. eq I-6) by

and

$$
\nu^{\prime}=\left[\begin{array}{c}
\cos \psi \\
-\sin \psi \sin (\theta / 2) \\
-\sin \psi \cos (\theta / 2)
\end{array}\right]
$$

$$
\begin{aligned}
& \nu=\left[\begin{array}{c}
\cos \omega \\
\sin \omega \sin (\theta / 2) \\
-\sin \omega \cos (\theta / 2)
\end{array}\right] \\
& \text { in the } X Y Z \text { system }
\end{aligned}
$$

According to eq I-23 we have

$$
\begin{aligned}
\mathbf{V}(\psi, \omega)= & {\left[1,\left(\sin ^{2} \psi+\sin ^{2} \omega\right) \cos ^{2}(\theta / 2),\right.} \\
& \sin ^{2} \psi \sin ^{2} \omega \cos ^{4}(\theta / 2) \\
& (\cos \psi \cos \omega+\cos \theta \sin \psi \sin \omega)^{2}, \\
& (\cos \psi \cos \omega+\cos \theta \sin \psi \sin \omega) \\
& \left.\times \sin \psi \sin \omega \cos ^{2}(\theta / 2)\right]
\end{aligned}
$$

Substitution of $\mathbf{V}(\psi, \omega)$ into eq I-32 (or eq 16 below) gives the required intensity function $I(\psi, \omega)$.

We are interested in the information as maximum as obtainable from light scattering measurements. We reasonably expect that there exists some limit to the information obtained by changing $\psi$ and $\omega$, though there are infinite combinations of $\psi$ and $\omega$. In this connection the following relation is instructive.

$$
\mathbf{V}(\psi, \omega)=\tilde{a} \mathbf{V}_{\mathrm{VV}_{\mathrm{V}}}+\tilde{b} \mathbf{V}_{\mathrm{HV}}+\tilde{c} \mathbf{V}_{\mathrm{Hh}}+\tilde{d} \mathbf{V}\left(45^{\circ}, 45^{\circ}\right)
$$

where $\mathbf{V}_{\mathrm{V}_{\mathrm{V}}}, \mathbf{V}_{\mathrm{HV}}\left[\equiv \mathbf{V}\left(0^{\circ}, 90^{\circ}\right)\right]$, and $\mathbf{V}_{\mathrm{Hh}}$ are given by eq I-34-36, and

$$
\begin{aligned}
\mathbf{V}\left(45^{\circ}, 45^{\circ}\right)= & {\left[1, \frac{1}{2}(1+\cos \theta), \frac{1}{16}(1+\cos \theta)^{2},\right.} \\
& \left.\frac{1}{4}(1+\cos \theta)^{2}, \frac{1}{8}(1+\cos \theta)^{2}\right]
\end{aligned}
$$

$\tilde{a}=\cos \psi \cos \omega \cos (\psi+\omega)$

$\tilde{b}=\sin ^{2} \psi+\sin ^{2} \omega-2 \sin \psi \sin \omega \cos (\psi-\omega)$

$\tilde{c}=-\sin \psi \sin \omega \cos (\psi+\omega)$

$\tilde{d}=\sin 2 \phi \sin 2 \omega$

Hence we have

$$
I(\phi, \omega)=\tilde{a} I_{\mathrm{Vv}}+\tilde{b} I_{\mathrm{Hv}}+\tilde{c} I_{\mathrm{Hh}}+\tilde{d} I\left(45^{\circ}, 45^{\circ}\right)
$$

where $I_{\mathrm{Vv}}, I_{\mathrm{Hv}}$, and $I_{\mathrm{Hh}}$ are given by eq I-37-39, and $I\left(45^{\circ}, 45^{\circ}\right)$ is obtained by substituting eq 4 into eq I-32 (or eq 16 below).

Equation 6 indicates that measurements at the four combinations of $\psi$ and $\omega$, implied at the rhs of eq 6 , are sufficient; measurements at additional angle combinations are redundant. Instead of $\phi=\omega=45^{\circ}$ we can use any other $\psi^{\prime}$ and $\omega^{\prime}$ if $\mathbf{V}\left(\psi^{\prime}, \omega^{\prime}\right)$ is independent of $\mathbf{V}_{\mathrm{VV}_{\mathrm{v}}}, \mathbf{V}_{\mathrm{HV}}$, and $\mathbf{V}_{\mathrm{Hh}}$, or unless it is expressible as a linear combination of the latter. In this case $\tilde{a}-\tilde{d}$ in eq 5 are varied of course. If, for example, $\psi=-\omega=45^{\circ}$ is used, the sign of $\omega$ in $\tilde{a}-\tilde{d}$ is to be changed. We have chosen $\psi=\omega=45^{\circ}$ above because $V\left(45^{\circ}, 45^{\circ}\right)$ has non-vanishing elements around $\theta=0$ ( $c f$. eq 4$)$ as well as $\tilde{a}-\tilde{d}$ 
Out-of-Plane Light Scattering by an Isotropic System

assume simple functional forms.

$I\left(45^{\circ}, 45^{\circ}\right)$ and $R\left(45^{\circ}, 45^{\circ}\right)$

The foregoing analysis indicates that $I_{\mathrm{Vv}}, I_{\mathrm{HV}}$, $I_{\mathrm{Hh}}$, and $I\left(45^{\circ}, 45^{\circ}\right)$ are of particular importance if studied all together. The first three were fully worked out in I for a general polymer chain and the Porod-Kratky chain. In this paper, therefore, we treat $I\left(45^{\circ}, 45^{\circ}\right)$ and also $R\left(45^{\circ}\right.$, $\left.45^{\circ}\right)$.

From eq I-32 and eq 4 we have

$$
\begin{aligned}
I\left(45^{\circ}, 45^{\circ}\right)= & (1024)^{-1} \sum_{i, j}\left\langle\left[\left(11 F_{0}-46 F_{1}-21 F_{2}\right)(\operatorname{Tr} \gamma)\left(\operatorname{Tr} \gamma^{\prime}\right)+\left(-23 F_{0}+6 F_{1}+105 F_{2}\right)\left[(\operatorname{Tr} \gamma) \mathbf{r}^{T} \gamma^{\prime} \mathbf{r}\right.\right.\right. \\
& \left.+\left(\operatorname{Tr} \gamma^{\prime}\right) \mathbf{r}^{T} \gamma \mathbf{r}\right] r^{-2}-21\left(3 F_{0}-30 F_{1}+35 F_{2}\right)\left(\mathbf{r}^{T} \gamma \mathbf{r}\right)\left(\mathbf{r}^{T} \gamma^{\prime} \mathbf{r}\right) r^{-4}+2\left(43 F_{0}+50 F_{1}-21 F_{2}\right) \operatorname{Tr} \gamma \gamma^{\prime} \\
& +4\left(25 F_{0}-138 F_{1}+105 F_{2}\right)\left(\mathbf{r}^{T} \gamma \gamma^{\prime} \mathbf{r}\right) r^{-2}+2\left\{\left(15 F_{0}+42 F_{1}-17 F_{2}\right)(\operatorname{Tr} \gamma)\left(\operatorname{Tr} \gamma^{\prime}\right)\right. \\
& +\left(21 F_{0}-114 F_{1}+85 F_{2}\right)\left[(\operatorname{Tr} \gamma) \mathbf{r}^{T} \gamma^{\prime} \mathbf{r}+\left(\operatorname{Tr} \gamma^{\prime}\right) \mathbf{r}^{T} \gamma \mathbf{r}\right] r^{-2} \\
& -17\left(3 F_{0}-30 F_{1}+35 F_{2}\right)\left(\mathbf{r}^{T} \gamma \mathbf{r}\right)\left(\mathbf{r}^{T} \gamma^{\prime} \mathbf{r}\right) r^{-4}+2\left(15 F_{0}-22 F_{1}-17 F_{2}\right) \operatorname{Tr} \gamma \gamma^{\prime} \\
& \left.-4\left(11 F_{0}+18 F_{1}-85 F_{2}\right)\left(\mathbf{r}^{T} \gamma \gamma^{\prime} \mathbf{r}\right) r^{-2}\right\} \cos \theta+\left\{\left(35 F_{0}-30 F_{1}+3 F_{2}\right)(\operatorname{Tr} \gamma)\left(\operatorname{Tr} \gamma^{\prime}\right)\right. \\
& -3\left(5 F_{0}-18 F_{1}+5 F_{2}\right)\left[(\operatorname{Tr} \gamma) \mathbf{r}^{T} \gamma^{\prime} \mathbf{r}+\left(\operatorname{Tr} \gamma^{\prime}\right) \mathbf{r}^{T} \gamma \mathbf{r}\right] r^{-2} \\
& +3\left(3 F_{0}-30 F_{1}+35 F_{2}\right)\left(\mathbf{r}^{T} \gamma \mathbf{r}\right)\left(\mathbf{r}^{T} \boldsymbol{r}^{\prime} \mathbf{r}\right) r^{-4}+2\left(3 F_{0}+2 F_{1}+3 F_{2}\right) \operatorname{Tr} \gamma \gamma^{\prime} \\
& \left.\left.\left.+4\left(F_{0}+6 F_{1}-15 F_{2}\right)\left(\mathbf{r}^{T} \gamma \gamma^{\prime} \mathbf{r}\right) r^{-2}\right\} \cos ^{2} \theta\right]_{i j}\right\rangle
\end{aligned}
$$

The series (in $s k$ ) expression of $I\left(45^{\circ}, 45^{\circ}\right)$ can be obtained from eq 4 and I-46 (or eq 21 below).

The reduced intensity for a dilute polymer solution of a general polymer chain is expressed

$$
\begin{aligned}
& R\left(45^{\circ}, 45^{\circ}\right)=\frac{1}{4} K c M_{\bar{\gamma}^{2}}\left[\frac{13}{4} f_{2}-f_{9}(s k)^{2}+\cdots+\sum_{i, i}\left\langle\left[\bar{\gamma} \bar{\gamma}^{\prime} F_{0}\right]_{i j}\right\rangle\right. \\
& \left.\quad+2\left\{\frac{1}{4} f_{2}-f_{10}(s k)^{2}+\cdots+\sum_{i, j}\left\langle\left[\bar{\gamma} \bar{\gamma}^{\prime} F_{0}\right]_{i j}\right\rangle\right\} \cos \theta+\left\{\frac{1}{4} f_{2}-f_{8}(s k)^{2}+\cdots+\sum_{i, j}\left\langle\left[\bar{\gamma} \bar{\gamma}^{\prime} F_{0}\right]_{i j}\right\rangle\right\} \cos ^{2} \theta\right] \\
& f_{9}=\frac{1}{210} \sum_{i<j}\left\langle\left[-\frac{133}{9} r^{2}(\operatorname{Tr} \gamma)\left(\operatorname{Tr} \gamma^{\prime}\right)+7(\operatorname{Tr} \gamma) \mathbf{r}^{T} \gamma^{\prime} \mathbf{r}+7\left(\operatorname{Tr} \gamma^{\prime}\right) \mathbf{r}^{T} \gamma \mathbf{r}+35 r^{2} \operatorname{Tr} \gamma \gamma^{\prime}-14 \mathbf{r}^{T} \gamma \gamma^{\prime} \mathbf{r}\right]_{i j}\right\rangle \\
& =\frac{1}{30} \sum_{i<j}\left\langle\left[\bar{\gamma} \mathbf{r}^{T} \hat{\gamma}^{\prime} \mathbf{r}+\bar{\gamma}^{\prime} \mathbf{r}^{T} \hat{\gamma} \mathbf{r}+5 r^{2} \operatorname{Tr} \hat{\gamma}^{\prime} \hat{\gamma}^{\prime}-2 \mathbf{r}^{T} \hat{\gamma} \hat{\gamma}^{\prime} \mathbf{r}\right]_{i j}\right\rangle \\
& f_{10}=\frac{1}{210} \sum_{i<j}\left\langle\left[\frac{11}{9} r^{2}(\operatorname{Tr} \gamma)\left(\operatorname{Tr} \gamma^{\prime}\right)-3(\operatorname{Tr} \gamma) \mathbf{r}^{T} \gamma^{\prime} \mathbf{r}-3\left(\operatorname{Tr} \gamma^{\prime}\right) \mathbf{r}^{T} \gamma \mathbf{r}-3 r^{2} \operatorname{Tr} \gamma \gamma^{\prime}+16 \mathbf{r}^{T} \gamma \gamma^{\prime} \mathbf{r}\right]_{i j}\right\rangle \\
& =\frac{1}{210} \sum_{i<j}\left\langle\left[7 \bar{\gamma} \mathbf{r}^{T} \hat{\gamma}^{\prime} \mathbf{r}+7 \bar{\gamma}^{\prime} \mathbf{r}^{T} \hat{\gamma} \mathbf{r}-3 r^{2} \operatorname{Tr} \hat{\gamma} \hat{\gamma}^{\prime}+16 \mathbf{r}^{T} \hat{\gamma} \hat{\gamma}^{\prime} \mathbf{r}\right]_{i j}\right\rangle
\end{aligned}
$$

For the Porod-Kratky chain we have

$$
\begin{aligned}
R\left(45^{\circ}, 45^{\circ}\right)= & \frac{1}{4} K c M\left(\frac{26}{135} x^{-1} \varepsilon^{2} g_{2}-\frac{2}{135} a^{2} \varepsilon(s k)^{2} g_{9}+\cdots+\left[1-\frac{1}{9} a t(s k)^{2} g_{1}+\cdots\right]\right. \\
+ & 2\left\{\frac{2}{135} x^{-1} \varepsilon^{2} g_{2}-\frac{2}{135} a^{2} \varepsilon(s k)^{2} g_{10}+\cdots+\left[1-\frac{1}{9} a t(s k)^{2} g_{1}+\cdots\right]\right\} \cos \theta \\
+ & \left.\left\{\frac{2}{135} x^{-1} \varepsilon^{2} g_{2}-\frac{2}{135} a^{2} \varepsilon(s k)^{2} g_{8}+\cdots+\left[1-\frac{1}{9} a t(s k)^{2} g_{1}+\cdots\right]\right\} \cos ^{2} \theta\right] \\
g_{9}= & 1-\frac{1}{9}(24-5 \varepsilon) \frac{1}{x}+\frac{1}{27}(78-22 \varepsilon) \frac{1}{x^{2}}-\frac{1}{20}(60-19 \varepsilon) \frac{1}{x^{2}} \mathrm{e}^{-x} \\
& +\frac{1}{108}(12-17 \varepsilon) \frac{1}{x^{2}} \mathrm{e}^{-3 x}+\frac{1}{18} \frac{\varepsilon}{x} \mathrm{e}^{-3 x}+\frac{1}{45} \frac{\varepsilon}{x^{2}} \mathrm{e}^{-6 x}
\end{aligned}
$$




\section{K. NAGAI}

$$
\begin{aligned}
g_{10}= & 1-\frac{1}{126}(336-31 \varepsilon) \frac{1}{x}+\frac{1}{756}(2184-295 \varepsilon) \frac{1}{x^{2}}-\frac{1}{2}(6-\varepsilon) \frac{1}{x^{2}} \mathrm{e}^{-x} \\
& +\frac{1}{378}(42-43 \varepsilon) \frac{1}{x^{2}} \mathrm{e}^{-3 x}-\frac{4}{63} \frac{\varepsilon}{x} \mathrm{e}^{-3 x}+\frac{1}{252} \frac{\varepsilon}{x^{2}} \mathrm{e}^{-6 x}
\end{aligned}
$$

If in eq 11 the series are truncated at $(s k)^{2}$ and the higher order (in $x^{-1}$ ) terms than $x^{-1}$ and the exponential terms are ignored, we have

$$
R\left(45^{\circ}, 45^{\circ}\right)=\frac{1}{4} K c M\left[\frac{8}{45} x^{-1} \varepsilon^{2}+\left\{1+\frac{2}{135} x^{-1} \varepsilon^{2}-\frac{1}{9} a t\left(1-\frac{3}{x}+\frac{2}{15} \frac{\varepsilon}{x}\right)(s k)^{2}\right\}(1+\cos \theta)^{2}\right]
$$

which can be rearranged to

$$
\left[\frac{4 R\left(45^{\circ}, 45^{\circ}\right)}{K c M}-\frac{8}{45} \frac{\varepsilon^{2}}{x}\right](1+\cos \theta)^{-2}=1+\frac{2}{135} \frac{\varepsilon^{2}}{x}-\frac{1}{9} a t\left(1-\frac{3}{x}+\frac{2}{15} \frac{\varepsilon}{x}\right)(s k)^{2}
$$

In I we proposed a method for determining the Porod-Kratky chain parameters, based on the approximate expressions eq I-94-96 for $R_{\mathrm{VV}}, R_{\mathrm{HV}}$, and $R_{\mathrm{Hh}}$, like eq 14 for $R\left(45^{\circ}, 45^{\circ}\right)$. We have recently noticed regretfully that a suggested plot of $R_{\mathrm{Hh}}$ against $\sin ^{2}(\theta / 2)$ may lead to errors for the following reason. In eq I-97, an ignored term of order $(s k)^{4}$ in $R_{\mathrm{Hh}}$, when divided by $(s k)^{2}$, becomes the same order of magnitude as the second term of the rhs, with respect to $\sin ^{2}(\theta / 2)$, and hence may affect the slope of the plot (but does not affect the intercept). No such difficulty occurs with a plot as implied in eq 15 , and hence $R\left(45^{\circ}, 45^{\circ}\right)$ is preferred and recommended over $R_{\mathrm{Hh}}$.

\section{Refined Method for Analysis of Experimental Data}

Addition of the $I\left(45^{\circ}, 45^{\circ}\right)$ expression to the previously obtained $I_{\mathrm{Vv}}, I_{\mathrm{HV}}$, and $I_{\mathrm{Hh}}$ expressions provides us a refined method for analysis of experimental data. Initially we wish to modify and supplement some previous results. ${ }^{1}$

In most cases intensity expressions are simplified if the polarizability tensor $\boldsymbol{\gamma}_{i}$ is divided into the anisotropic and isotorpic parts: $\boldsymbol{\gamma}_{i}=\hat{\boldsymbol{\gamma}}_{i}+\bar{\gamma}_{i} \mathbf{E}_{3}$. If this is done, eq I-32 becomes

where

$$
I=(64)^{-1} \sum_{i, j}\left\langle\left[\mathbf{V}\left(\mathbf{Q}_{0}{ }^{\prime} F_{0}+\mathbf{Q}_{1}{ }^{\prime} F_{1}+\mathbf{Q}_{2}{ }^{\prime} F_{2}\right) \mathbf{U}^{\prime \prime}\right]_{i j}\right\rangle
$$

$$
\begin{aligned}
& \mathbf{Q}_{0}{ }^{\prime}=\left[\begin{array}{rrrrr}
0 & 0 & 3 & 2 & 12 \\
0 & 0 & -15 & 6 & -12 \\
0 & 0 & 105 & 6 & -60 \\
64 & 16 & 6 & 4 & -8 \\
0 & -48 & -60 & -8 & 48
\end{array}\right] \\
& \mathbf{Q}_{1}{ }^{\prime}=\left[\begin{array}{rrrrr}
0 & 0 & -30 & 12 & -24 \\
0 & 0 & 150 & -12 & -24 \\
0 & 0 & -1050 & -60 & 600 \\
0 & -48 & -60 & -8 & 48 \\
0 & 144 & 600 & 48 & -384
\end{array}\right] \\
& \mathbf{Q}_{2}{ }^{\prime}=\left[\begin{array}{rrrrr}
0 & 0 & 35 & 2 & -20 \\
0 & 0 & -175 & -10 & 100 \\
0 & 0 & 1225 & 70 & -700 \\
0 & 0 & 70 & 4 & -40 \\
0 & 0 & -700 & -40 & 400
\end{array}\right]
\end{aligned}
$$


Out-of-Plane Light Scattering by an Isotropic System

$$
\mathbf{U}^{\prime \prime}=\left[\begin{array}{c}
\bar{\gamma} \bar{\gamma}^{\prime} \\
r^{-2}\left(\bar{\gamma} \mathbf{r}^{T} \hat{\gamma}^{\prime} \mathbf{r}+\bar{\gamma}^{\prime} \mathbf{r}^{T} \hat{\gamma} \mathbf{r}\right) \\
r^{-4}\left(\mathbf{r}^{T} \hat{\gamma} \mathbf{r}\right)\left(\mathbf{r}^{T} \hat{\gamma} \mathbf{r}\right) \\
\operatorname{Tr} \hat{\gamma} \hat{\gamma}^{\prime} \\
r^{-2} \mathbf{r}^{T} \hat{\gamma} \hat{\gamma}^{\prime} \mathbf{r}
\end{array}\right]
$$

Similarly eq I-46 becomes

$$
\begin{gathered}
I=2^{-1} \sum_{i, j} \sum_{k=0}^{\infty}\left\langle\left[(-1)^{k}[(2 k+1) !(2 k+3)(2 k+5)]^{-1}(k s r)^{2 k} \mathbf{V} \mathbf{W}_{k}{ }^{\prime} \mathbf{U}^{\prime \prime}\right]_{i j}\right\rangle \\
\mathbf{W}_{k}{ }^{\prime}=\left[\begin{array}{ccrcc}
0 & 0 & k(k-1) & 2 k^{2}+6 k+3 & -4 k(k+2) \\
0 & 0 & -5 k(k-1) & -2 k(k+2) & 2 k(4 k+5) \\
0 & 0 & 35 k(k-1) & 2 k(k-1) & -20 k(k-1) \\
8 k^{2}+32 k+30 & -2 k(2 k+5) & 2 k(k-1) & 1 & 4 k \\
0 & 6 k(2 k+5) & -20 k(k-1) & 4 k & 4 k(2 k-5)
\end{array}\right]
\end{gathered}
$$

Let us define

$$
\begin{aligned}
& \mathbf{V}_{1}=\left(\begin{array}{lllll}
1 & 0 & 0 & 0 & 0
\end{array}\right) \\
& V_{2}=\left(\begin{array}{lllll}
0 & 1 & 0 & 0 & 0
\end{array}\right) \\
& \left.\begin{array}{l}
\cdot \cdots \\
\mathbf{V}_{5}=\left(\begin{array}{lllll}
0 & 0 & 0 & 0 & 1
\end{array}\right)
\end{array}\right\}
\end{aligned}
$$

and the corresponding $I_{1}, I_{2}, \ldots, I_{5}$ and express these $I_{i}$ in the vectorial expression

$$
\mathrm{I}=\left(\begin{array}{lllll}
I_{1} & I_{2} & I_{3} & I_{4} & I_{5}
\end{array}\right)^{T}
$$

Obviously $I$ is nothing but $I$ in eq 16 or 21 with the factor $\mathbf{V}$ omitted. We further define $I_{e}$, using the new quantity $I\left(45^{\circ}, 45^{\circ}\right)$ :

$$
\mathbf{I}_{\mathrm{e}}=\left[I_{\mathrm{Vv}} I_{\mathrm{Hv}} I_{\mathrm{Hh}} I\left(45^{\circ}, 45^{\circ}\right)\right]^{T}
$$

Then we have

$$
\Theta=\left[\begin{array}{ccccc}
1 & 0 & 0 & 1 & 0 \\
1 & \frac{1}{2}(1+c) & 0 & 0 & 0 \\
1 & 1+c & \frac{1}{4}(1+c)^{2} & \mathbf{c}^{2} & \frac{1}{2} \mathrm{c}(1+\mathrm{c}) \\
1 & \frac{1}{2}(1+\mathrm{c}) & \frac{1}{16}(1+\mathrm{c})^{2} & \frac{1}{4}(1+\mathrm{c})^{2} & \frac{1}{8}(1+\mathrm{c})^{2}
\end{array}\right]_{\theta}
$$

where the subscript $\theta$ is implied to apply to c, yielding $\cos \theta$.

We note that for a quite general model the $I_{i}$ 's are mutually independent, or any of them cannot be expressed as a linear combination of the remainders. (We have not yet been aware of any rigorous proof for this statement, but it appears almost valid.) Therefore it is desirable but impossible to determine $I_{i}$ experimentally, because the unknowns are five whereas the independent observables at most are four. We observe from eq 16-24 that the isotropic-scattering term

$$
I \text { (iso) }=\sum_{i, j}\left\langle\left[\bar{\gamma} \bar{\gamma}^{\prime} F_{0}\right]_{i j}\right\rangle
$$

is included in $I_{4}$. We have no means of separating it from the anisotropic-scattering term. The 
conclusions of this paragraph are restricted to the perfect intensity functions for a quite general model. With approximate forms for the intensity functions, or for special models, there remain possibilities for separating the $I_{i}$ 's as well as for separating the isotropic-scattering term. For example, Utiyama and Kurata ${ }^{3}$ showed that the isotropic- and anisotropic-scattering terms can be separated rigorously by using, $I_{\mathrm{V}_{\mathrm{v}}}, I_{\mathrm{HV}}$, and $I_{\mathrm{Hh}}$, for the the random chain. This result becomes true for any model if the series in eq 21 is truncated at $(s k)^{2}$, as shown below.

We observe that the third rows and columns of $\mathbf{W}_{0}{ }^{\prime}$ and $\mathbf{W}_{1}{ }^{\prime}$ are zero. Hence, after the truncation at $(s k)^{2} I_{3}$ is identically zero and the term $r^{-4}\left(\mathbf{r}^{T} \hat{\gamma} \mathbf{r}\right)\left(\mathbf{r}^{T} \hat{\gamma}^{\prime} \mathbf{r}\right)$ in eq 20 contributes nothing. Deleting the vanishing third rows and columns in $\mathbf{W}_{0}^{\prime}$ and $\mathbf{W}_{1}^{\prime}$ we have

$$
\begin{aligned}
\tilde{\mathbf{I}} & =\left(I_{1} I_{2} I_{4} I_{5}\right)^{T}=\widetilde{\mathbf{I}}^{(0)}-\widetilde{\mathbf{I}}^{(1)}(s k)^{2} \\
& =\frac{1}{30} \widetilde{\mathbf{W}}_{0}\left[\begin{array}{c}
\bar{\gamma}^{2} \\
0 \\
\left\langle\operatorname{Tr} \hat{\gamma}^{2}\right\rangle \\
0
\end{array}\right]-\frac{1}{210} \widetilde{\mathbf{W}}_{1} \mathbf{S}(s k)^{2}
\end{aligned}
$$

where

$$
\begin{aligned}
& \tilde{\mathbf{I}}^{(k)}=\left(I_{1}^{(k)} I_{2}{ }^{(k)} I_{4}{ }^{(k)} I_{5}{ }^{(k)}\right)^{T}, \quad k=0,1 \\
& \widetilde{\mathbf{W}}_{k}=\left[\begin{array}{cccc}
0 & 0 & 2 k^{2}+6 k+3 & -4 k(k+2) \\
0 & 0 & -2 k(k+2) & 2 k(4 k+5) \\
8 k^{2}+32 k+30 & -2 k(2 k+5) & 1 & 4 k \\
0 & 6 k(2 k+5) & 4 k & 4 k(2 k-5)
\end{array}\right], \quad k=0,1 \\
& \mathbf{S}=\left(S_{1} S_{2} S_{4} S_{5}\right)^{T} \\
& S_{1}=\sum_{i<j}\left\langle\left[\bar{\gamma} \bar{\gamma}^{\prime} r^{2}\right]_{i j}\right\rangle \\
& \boldsymbol{S}_{2}=\sum_{i<j}\left\langle\left[\bar{\gamma}^{\mathbf{T}} \hat{\gamma}^{\prime} \mathbf{r}+\bar{\gamma}^{\prime} \mathbf{r}^{T} \hat{\gamma} \mathbf{r}\right]_{i j}\right\rangle \\
& S_{4}=\sum_{i<j}\left\langle\left[r^{2} \operatorname{Tr} \hat{\gamma} \hat{\gamma}^{\prime}\right]_{i j}\right\rangle \\
& S_{5}=\sum_{i<j}\left\langle\left[\mathbf{r}^{T} \hat{\gamma} \hat{\gamma}^{\prime} \mathbf{r}\right]_{i j}\right\rangle
\end{aligned}
$$

Comparing the coefficients of $(s k)^{0}$ in eq 29 and 30 , we have

$$
\tilde{\mathbf{I}}^{(0)}=\frac{1}{30} \tilde{\mathbf{W}}_{0}\left[\begin{array}{c}
\bar{\gamma}^{2} \\
0 \\
\left\langle\operatorname{Tr} \hat{\boldsymbol{\gamma}}^{2}\right\rangle \\
0
\end{array}\right]=\frac{1}{30}\left[\begin{array}{c}
3\left\langle\operatorname{Tr} \hat{\gamma}^{2}\right\rangle \\
0 \\
30 \bar{\gamma}^{2}+\left\langle\operatorname{Tr} \hat{\gamma}^{2}\right\rangle \\
0
\end{array}\right]
$$

and therefrom

$$
\begin{gathered}
\bar{\gamma}^{2}=I_{4}{ }^{(0)}-\frac{1}{3} I_{1}{ }^{(0)} \\
\left\langle\operatorname{Tr} \hat{\gamma}^{2}\right\rangle=10 I_{1}{ }^{(0)}
\end{gathered}
$$

From the coefficients of $(s k)^{2}$ we have

$$
\mathbf{S}=210 \widetilde{\mathbf{W}}_{1}^{-1} \tilde{\mathbf{I}}^{(1)}
$$

with 
Out-of-Plane Light Scattering by an Isotropic System

$$
\widetilde{\mathbf{W}}_{1}^{-1}=\frac{1}{630}\left[\begin{array}{rrrr}
-3 & -2 & 9 & 3 \\
0 & 10 & 0 & 15 \\
90 & 60 & 0 & 0 \\
30 & 55 & 0 & 0
\end{array}\right]
$$

Equation 26 now reads $\tilde{\mathbf{I}}_{\mathrm{e}}=\tilde{\Theta} \tilde{\mathbf{I}}$ or

$$
\tilde{\mathbf{I}}=\widetilde{\Theta}^{-1} \widetilde{\mathbf{I}}_{\mathrm{e}}
$$

where $\widetilde{\Theta}$ is a matrix formed from $\Theta$ by deleting its third column, and its inverse is calculated as

$$
\tilde{\Theta}^{-1}=\left[\sin ^{2} \theta(1+\cos \theta)\right]^{-1}\left[\begin{array}{crrc}
-c(1+c)^{2} & 2\left(1-c^{2}\right) & -(1+c)^{2} & 4 c(1+c) \\
+2 c(1 c) & -2(1-c)^{2} & 2(1+c) & -8 c \\
(1+c)^{2} & -2\left(1-c^{2}\right) & (1+c)^{2} & -4 c(1+c) \\
-2(1+c)^{2} & -4(1-c)^{2} & -2(1+c)^{2} & 8\left(1+c^{2}\right)
\end{array}\right]_{\theta}
$$

$\tilde{\mathbf{I}}$ is calculated from the observed $\mathbf{I}_{e}$ according to eq $40 . \quad I_{i}{ }^{(0)}$ and $I_{i}{ }^{(1)}$ are obtained as the intercept and slope in the plot of $I_{i}$ against $(s k)^{2}$. The linearity of these plots and the requirement $I_{2}{ }^{(0)}=I_{5}{ }^{(0)}=0$ (cf. eq 35) serve as a criterion for the truncatability of the series in eq 21 at $(s k)^{2}$. From $\widetilde{\mathbf{I}}^{(0)}$ and $\widetilde{\mathbf{I}}^{(1)}$ thus determined we can obtain $\bar{\gamma}^{2},\left\langle\operatorname{Tr} \hat{\gamma}^{2}\right\rangle$, and $\mathbf{S}$. Thus the $\mathbf{I}_{i}$ 's are separable and $I($ iso $)=\bar{\gamma}^{2}-(s k)^{2} S_{1} / 3$ is extractable experimentally with the aid of the theory.

Like $\widetilde{\mathbf{I}}, \mathbf{I}_{\mathrm{e}}$ has to be expressible

$$
\mathbf{I}_{\mathrm{e}}=\mathbf{I}_{\mathrm{e}}^{(0)}-\mathbf{I}_{\mathrm{e}}^{(1)}(s k)^{2}
$$

$\mathbf{I}_{e}^{(0)}$ and $\mathbf{I}_{e}^{(1)}$ could be used instead of $\tilde{\mathbf{I}}^{(0)}$ and $\tilde{\mathbf{I}}^{(1)}$ to express $\bar{\gamma}^{2},\left\langle\operatorname{Tr} \hat{\boldsymbol{\gamma}}^{2}\right\rangle$, and $\mathbf{S}$, and would be preferred because $\mathbf{I}_{\theta}$ is more directly accessible experimentally than $\tilde{\mathbf{I}}$. A difficulty occurs in this procedure, however, in that the determination of $\mathbf{I}_{e}{ }^{(0)}$ and $\mathbf{I}_{e}{ }^{(1)}$ through eq 42 is not easy because most of their elements are dependent on $\theta$. The exception is the first element of $\mathbf{I}_{\mathrm{e}}$, i.e., $I_{\mathrm{Vv}}=I_{\mathrm{V}_{\mathrm{v}}}^{(0)}-I_{\mathrm{Vv}}^{(1)}(s k)^{2}$ where both $I^{(0)}{ }_{\mathrm{v}}$ and $I^{(1)}{ }_{\mathrm{V} v}$ are independent of $\theta$; clearly these two are not sufficient for the purpose in hand. Multiplication of $\tilde{\boldsymbol{\Theta}}^{-1}$ to $\mathbf{I}_{\mathrm{e}}$, which yields $\tilde{\mathbf{I}}$, has eliminated the $\theta$ dependence of $\mathbf{I}_{\mathrm{e}}{ }^{(0)}$ and $\mathbf{I}_{\mathrm{e}}{ }^{(1)}$.

The foregoing results are readily transcribable to those for dilute polymer solutions; it suffices to replace $\mathbf{I}_{\mathrm{e}}$ and $\tilde{\mathbf{I}}$ with $\mathbf{R}_{\mathrm{e}}$ and $\tilde{\mathbf{R}}$ both multiplied with $\bar{\gamma}^{2} /(K c M)$, where

$$
\mathbf{R}_{\mathrm{e}}=\left[\boldsymbol{R}_{\mathrm{Vv}} \boldsymbol{R}_{\mathrm{Hv}} R_{\mathrm{Hh}} R\left(45^{\circ}, 45^{\circ}\right)\right]^{T}
$$

$$
\begin{gathered}
\tilde{\mathbf{R}}=\tilde{\Theta}^{-1} \mathbf{R}_{\mathrm{e}}=\widetilde{\mathbf{R}}^{(0)}-\tilde{\mathbf{R}}^{(1)}(s k)^{2} \\
\tilde{\mathbf{R}}^{(k)}=\left(\boldsymbol{R}_{1}{ }^{(k)}{R_{2}}^{(k)} R_{4}{ }^{(k)} R_{5}{ }^{(k)}\right)^{T}, \quad k=0,1
\end{gathered}
$$

After this replacement eq $36-38$ change to

$$
\begin{aligned}
& M=\left({R_{4}}^{(0)}-\frac{1}{3} R_{1}{ }^{(0)}\right) /(K c) \\
& \bar{\gamma}^{-2}\left\langle\operatorname{Tr} \hat{\gamma}^{2}\right\rangle=10 R_{1}{ }^{(0)} /(K c M) \\
& \bar{\gamma}^{-2} \mathbf{S}=210 \widetilde{\mathbf{W}}_{1}^{-1} \widetilde{\mathbf{R}}^{(1)} /(K c M)
\end{aligned}
$$

Therefore we can obtain the molecular weight $M$, the mean optical anisotropy $(3 / 2)\left\langle\operatorname{Tr} \hat{\gamma}^{2}\right\rangle$, and $S$ from experimental data through eq $46-48$. In the case of dilute polymer solutions, $\bar{\gamma}$, which now stands for the mean-excess polarizability, can be calculated from $M$ and the refractive index increment ( $c f$. eq I-49).

We would like to make some remarks on the foregoing results. First, $\mathbf{S}$ can be determined according to eq 38 or 48 without any specific assumption on the structure of a scattering system except that it is isotropic as a whole. Second, these equations are unsolvable without $I^{(1)}\left(45^{\circ}, 45^{\circ}\right)$ or $R^{(1)}\left(45^{\circ}, 45^{\circ}\right)$. Thus the out-ofplane scattering permits a more penetrating insight into the structure of a scattering system, and its importance is underscored.

A point not to be overlooked is as follows. The series in eq 21 can always be truncated at $(s k)^{2}$ for any system if $s k$ is selected as properly small. Therefore the method proposed above would be effective for any system. The statement is valid in principle, but not always so 


\section{K. NAGAI}

in practice. As is well known, measurements at very small angles are difficult, being hampered by the strong unscattered light. Another difficuly occurs for small $\theta$. The determinant of $\widetilde{\Theta}$ is zero and $\tilde{\Theta}^{-1}$ is indefinite at $\theta=0$. This indicates that the four elements of $I_{e}$ are not independent at $\theta=0$. As $\theta$ approaches zero, the elements of $\tilde{\Theta}^{-1}$ become very large while all elements of $\tilde{\mathbf{I}}$ and $\mathbf{I}_{e}$ remain finite. Hence, when $\widetilde{\Theta}^{-1}$ is multiplied to $I_{e}$ a serious cancellation is expected to occur and errors in $\mathbf{I}_{\boldsymbol{e}}$ are exaggerated in $\tilde{\mathbf{I}}$. Possible errors arising from this source will be judged by the scattering of data points in the plots of the elements of $\tilde{\mathbf{I}}$ against $(s k)^{2}$. The above arguments about $\mathbf{I}_{e}$ and $\tilde{\mathbf{I}}$ can be applied to $\mathbf{R}_{\mathrm{e}}$ and $\widetilde{\mathbf{R}}$ as well of course.

$S_{i}$ for the Porod-Kratky chain can be calculated in a manner previously described ${ }^{1}$ :

$$
\begin{aligned}
S_{1}= & \frac{1}{3} \bar{\alpha}^{2} a t^{3}\left(1-\frac{3}{x}+\frac{6}{x^{2}}-\frac{6}{x^{3}}+\frac{6}{x^{3}} \mathrm{e}^{-x}\right) \\
S_{2}= & \frac{4}{9} \bar{\alpha} \Delta \alpha a^{2} t^{2}\left(1-\frac{8}{3 x}+\frac{26}{9 x^{2}}-\frac{3}{x^{2}} \mathrm{e}^{-x}\right. \\
& \left.+\frac{1}{9 x^{2}} \mathrm{e}^{-3 x}\right) \\
S_{4}= & \frac{2}{27}(\Delta \alpha)^{2} a^{3} t\left(1-\frac{3}{2 x}+\frac{9}{5 x} \mathrm{e}^{-x}-\frac{1}{3 x} \mathrm{e}^{-3 x}\right. \\
& \left.+\frac{1}{30 x} \mathrm{e}^{-6 x}\right) \\
S_{5}= & \frac{5}{81}(\Delta \alpha)^{2} a^{3} t\left(1-\frac{47}{30 x}+\frac{99}{50 x} \mathrm{e}^{-x}\right. \\
& \left.-\frac{13}{30 x} \mathrm{e}^{-3 x}-\frac{1}{5} \mathrm{e}^{-3 x}+\frac{1}{50 x} \mathrm{e}^{-6 x}\right)
\end{aligned}
$$

It is now possible to examine the validity of the Porod-Kratky chain as a model for real chains through the unapproximated forms of $S_{i}$. We wish to note the following point, however. A previous ${ }^{1}$ method for analyzing experimental data supplemented earlier in this paper ( $c f$. below eq 15), though approximate, has an advantage that results obtained paradoxically do not suffer from the Porod-Kratky chain model despite its use in the derivation of these results, as pointed out in DISCUSSION of $\mathrm{I}$, and hence are valid for any model of linear polymer chains.

\section{Anisotropic-Scattering Function in the Out-of- Plane Scattering}

Gouda and Prins ${ }^{2}$ introduced a $\mathrm{Hv}$ anisotropicscattering function $I_{\mathrm{HV}}^{\prime}$ in the out-of-plane scattering, which is defined for such an arrangement of the polarizer and the analyzer as $\nu^{\prime} \perp \nu$. They stated that this $I^{\prime}{ }_{\mathrm{HV}}$ is to be preferred over the ordinary $\mathrm{Hv}$ scattering $I_{\mathrm{HV}}$ (i.e., in the inplane scattering) because it includes more information. The condition $\nu^{\prime} \perp \nu$ reads according to eq 1

$$
\tan \omega=-(\cot \psi) / \cos \theta
$$

$\mathbf{V}_{\mathrm{HV}}^{\prime}$ related to $I_{\mathrm{HV}}^{\prime}$ becomes

$$
\begin{aligned}
\mathbf{V}_{H \mathrm{~V}}^{\prime}= & {\left[1,\left(\frac{1-\sin ^{4} \psi \sin ^{2} \theta}{1-\sin ^{2} \psi \sin ^{2} \theta}\right) \cos ^{2}(\theta / 2),\right.} \\
& \left.\left(\frac{\sin ^{2} \psi \cos ^{2} \psi}{1-\sin ^{2} \psi \sin ^{2} \theta}\right) \cos ^{4}(\theta / 2), 0,0\right]
\end{aligned}
$$

$I^{\prime}{ }_{\mathrm{HV}}$ involves more information than $I_{\mathrm{HV}}$, in accordance with Gouda and Prins, ${ }^{2}$ because it includes $I_{1}, I_{2}$, and $I_{3}$ whereas $I_{\mathrm{Hv}}$ includes only $I_{1}$ and $I_{2}$. If in eq 2 the fourth element of $\mathbf{V}(\psi, \omega)$ is forced to equal zero to eliminate the isotropic-scattering contribution, then the last element necessarily becomes zero. Therefore it is impossible to construct any anisotropic-scattering function which includes $I_{5}$ additionally; $I_{\mathrm{HV}}^{\prime}$ is the most general anisotropic-scattering function which can be defined with a single pair of $\phi$ and $\omega$. We wish to point out that nevertheless $I_{\mathrm{Hv}}^{\prime}$ can by no means include all information obtainable. This is accomplished only by studying all together the four intensity functions as included in eq 6.

\section{CASE OF PHOTOGRAPHIC DETECTION}

This method was initiated in Stein's laboratory ${ }^{4}$ and has been extensively used in his and other laboratories. The polarizer and the analyzer are set so that their optical axes are colinear, and the photographic plate is set vertical to the axes, as shown in Figure 2. Usually the vertically polarized incident light $\nu_{\mathrm{v}}{ }^{\prime}$ is used and the polarizer is set vertical $\left(\nu_{\mathrm{V}}\right.$, the $\mathrm{Vv}$ 


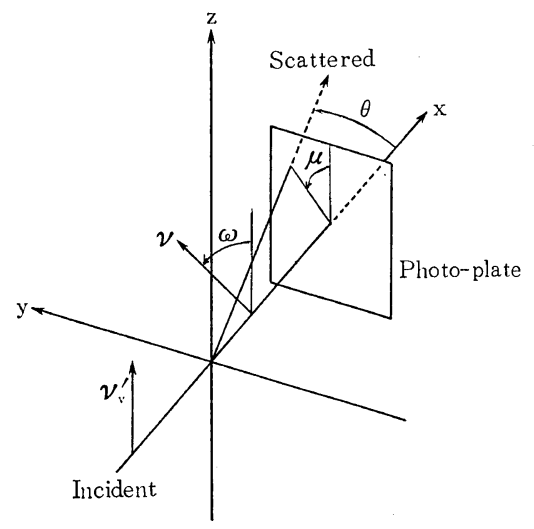

Figure 2.

pattern) or horizontal ( $\boldsymbol{\nu}_{\mathrm{H}}$, the Hv pattern).

As previously, we first consider the in-plane scattering in which the planes of polarization of the polarizer and the analyzer make angles $\phi$ and $\omega$ with the $z$ axis, similarly in Figure 1. In this case $\nu^{\prime}$ and $\nu$ are given by

$$
\nu^{\prime}=\left[\begin{array}{c}
\cos \psi \\
-\sin \phi \sin (\theta / 2) \\
-\sin \phi \cos (\theta / 2)
\end{array}\right]
$$

and

$$
\nu=\left[\begin{array}{c}
\cos \omega \\
-\sin \omega \sin (\theta / 2) \\
-\sin \omega \cos (\theta / 2)
\end{array}\right]
$$

in the $X Y Z$ system

$$
\text { (defined as in Figure 1) }
$$

Therefore we have according to eq I-23

$$
\begin{aligned}
\mathbf{V}= & {\left[1,\left(\sin ^{2} \psi+\sin ^{2} \omega\right) \cos ^{2}(\theta / 2),\right.} \\
& \sin ^{2} \psi \sin ^{2} \omega \cos ^{4}(\theta / 2), \cos ^{2}(\psi-\omega), \\
& \left.\cos (\psi-\omega) \sin \psi \sin \omega \cos ^{2}(\theta / 2)\right]
\end{aligned}
$$

The out-of-plane scattering at the direction of $\theta$ and the azimuthal angle $\mu$ while the polarizer and the analyzer making angles zero and $\omega$ with the $z$ axis, an arrangement shown in Figure 2, can be reduced to the in-plane scattering by rotating the scattering frameworkpolarizer, sample, analyzer, and photographic plate-about the $x$ axis by angle $90^{\circ}-\mu$. After this rotation the polarizer and the analyzer make angles $90^{\circ}-\mu$ and $90^{\circ}-\mu+\omega$, respectively, with the $z$ axis. Equating these to $\phi$ and $\omega$ in eq 53 we have

$$
\begin{aligned}
\mathbf{V}^{*}(\mu, \omega)= & {\left[1,\left(\cos ^{2} \mu+\cos ^{2}(\mu-\omega)\right) \cos ^{2}(\theta / 2),\right.} \\
& \cos ^{2} \mu \cos ^{2}(\mu-\omega) \cos ^{4}(\theta / 2), \cos ^{2} \omega, \\
& \left.\cos \mu \cos \omega \cos (\mu-\omega) \cos ^{2}(\theta / 2)\right]
\end{aligned}
$$

The corresponding intensity is obtained by substituting this $\mathrm{V}^{*}$ into eq I-32 or eq 16 . We find

$$
\begin{gathered}
\mathbf{V}^{*}(\mu, \omega)=\tilde{e} \mathbf{V}^{*}\left(\mu, 0^{\circ}\right)+\tilde{f} \mathbf{V}^{*}\left(\mu, 90^{\circ}\right) \\
+\tilde{g} \mathbf{V}^{*}\left(\mu, 45^{\circ}\right)
\end{gathered}
$$

where

$$
\begin{aligned}
\mathbf{V}^{*}\left(\mu, 0^{\circ}\right)= & {\left[1,2 \cos ^{2} \mu \cos ^{2}(\theta / 2),\right.} \\
& \left.\cos ^{4} \mu \cos ^{4}(\theta / 2), 1, \cos ^{2} \mu \cos ^{2}(\theta / 2)\right]
\end{aligned}
$$

$$
\begin{aligned}
\mathbf{V}^{*}\left(\mu, 90^{\circ}\right)= & {\left[1, \cos ^{2}(\theta / 2),\right.} \\
& \left.\cos ^{2} \mu \sin ^{2} \mu \cos ^{4}(\theta / 2), 0,0\right]
\end{aligned}
$$

$\mathbf{V}^{*}\left(\mu, 45^{\circ}\right)$

$$
\begin{aligned}
& =\left[1,\left(\frac{1}{2}+\cos ^{2} \mu+\cos \mu \sin \mu\right) \cos ^{2}(\theta / 2),\right. \\
& \left(\frac{1}{2}+\sin \mu \cos \mu\right) \cos ^{2} \mu \cos ^{4}(\theta / 2), \frac{1}{2}, \\
& \left.\frac{1}{2} \cos \mu(\cos \mu+\sin \mu) \cos ^{2}(\theta / 2)\right] \\
& \tilde{e}=\cos \omega(\cos \omega-\sin \omega) \\
& \tilde{f}=\sin \omega(\sin \omega-\cos \omega) \\
& \tilde{g}=\sin 2 \omega
\end{aligned}
$$

Therefore we have

$$
\begin{aligned}
I^{*}(\mu, \omega)= & \tilde{e} I^{*}\left(\mu, 0^{\circ}\right)+\tilde{f} I^{*}\left(\mu, 90^{\circ}\right) \\
& +\tilde{\boldsymbol{g}} I^{*}\left(\mu, 45^{\circ}\right)
\end{aligned}
$$

$I^{*}\left(\mu, 0^{\circ}\right)$ and $I^{*}\left(\mu, 90^{\circ}\right)$ are for the $\mathrm{Vv}$ and $\mathrm{Hv}$ patterns, respectively. Eq 60 indicates that the photograph for arbitrary $\mu$ and $\omega$ is composable from the three photographs as implied in the rhs of this equation.

All of the three photographs are not necessary for the purpose of obtaining the maximum information. We can readily confirm

$$
\begin{aligned}
& \mathbf{V}^{*}(\mu, \omega)=a^{*} \mathbf{V}^{*}\left(90^{\circ}, 0^{\circ}\right)+b^{*} \mathbf{V}^{*}\left(90^{\circ}, 90^{\circ}\right) \\
& \quad+c^{*} \mathbf{V}^{*}\left(0^{\circ}, 0^{\circ}\right)+d^{*} \mathbf{V}^{*}\left(45^{\circ}, 0^{\circ}\right)
\end{aligned}
$$

where 


\section{K. NAGAI}

$$
\left.\begin{array}{rl}
a^{*}= & -\sin \mu \sin (\mu-\omega) \cos (2 \mu-\omega) \\
b^{*}= & \cos ^{2} \mu+\cos ^{2}(\mu-\omega) \\
& -2 \cos \mu \cos \omega \cos (\mu-\omega) \\
c^{*}= & \cos \mu \cos (\mu-\omega) \cos (2 \mu-\omega) \\
d^{*}= & \sin 2 \mu \sin 2(\mu-\omega)
\end{array}\right\}
$$

Therefore we have

$$
\begin{aligned}
& I^{*}(\mu, \omega)=a^{*} I^{*}\left(90^{\circ}, 0\right)+b^{*} I^{*}\left(90^{\circ}, 90^{\circ}\right) \\
& \quad+c^{*} I^{*}\left(0^{\circ}, 0^{\circ}\right)+d^{*} I^{*}\left(45^{\circ}, 0^{\circ}\right)
\end{aligned}
$$

$I^{*}\left(90^{\circ}, 90^{\circ}\right)$ in the rhs of eq 63 is inclined in the $\mathrm{Hv}$ pattern, and the remaining three are included in the $\mathrm{Vv}$ pattern. Therefore the two photographs, i.e., the $\mathrm{Vv}$ and $\mathrm{Hv}$ patterns, are sufficient, including all information. The $\mathrm{Vv}$ and $\mathrm{Hv}$ patterns are symmetric with respect to $\mu=90^{\circ}$ and $45^{\circ}$, respectively, as is apparent from eq 56 and 57, a well-known experimental result. ${ }^{4}$ Any angle can be used in place of $45^{\circ}$ in eq 55 and 60, and in eq 61 and 63, unless it is zero or multiples of $90^{\circ}$.

It must be noted that the analysis of this section is valid only for small $\theta$, or with highest rigor only for $\theta=0$. For $\theta \neq 0$ the scattered beam passes the analyzer, inclining to its optical axis, so $\nu$ in eq 52 is invalidated. The necessary correction was given by van Aartsen and Keijzers, as quoted by Clough, van Aartsen, and Stein (Appendex A of ref. 5).

Acknowledgment. The author wishes to thank Prof. W. Prins of Syracuse University for his suggestion which stimulated this work.

\section{REFERENCES}

1. K. Nagai, Polymer J., 3, 67 (1972).

2. J. H. Gouda and W. Prins, J. Polym. Sci., Part $A-2,8,2029$ (1972).

3. H. Utiyama and M. Kurata, Bull. Ints. Chem. Res. Kyoto Univ., 42, 128 (1964).

4. R. S. Stein and M. B. Rhodes, J. Appl. Phys., 31, 1873 (1960).

5. S, Clough, J, J. van Aartsen, and R.S. Stein, J. Appl. Phys., 36, 3072 (1965). 